\title{
Porocarcinoma of the Left Arm: A Report of a Rare Skin Cancer Case and Literature Review
}

\author{
Carlo Signorelli ${ }^{1}$, Luca Zanella-Cavallero ${ }^{2}$, Valentina Ranucci ${ }^{3}$, Antonio Pellicciotti ${ }^{2}$, and \\ Enzo Ruggeri ${ }^{1}$ \\ ${ }^{1}$ Medical Oncology Unit, Belcolle Hospital, ASL Viterbo \\ ${ }^{2}$ General Surgery, Tarquinia Hospital, ASL Viterbo \\ ${ }^{3}$ Pathology Unit, Belcolle Hospital, ASL Viterbo
}

February 7, 2022

\begin{abstract}
Porocarcinoma is a rare type of skin cancer that develops from the intraepidermal ductal part of eccrine sweat glands. It can arise de novo or from poroma ground with high potential for locoregional metastatic spread, morbidity, and mortality. It mostly occurs in elderly patients.
\end{abstract}

\section{Introduction}

Porocarcinoma (PC), first described in 1963 by Pinkus and Mehregan, is a rare malignant skin tumor originating from the intraepidermal ductal portion of the sweat glands. It accounts for an estimated $0.005 \%-$ $0.01 \%$ of all cutaneous carcinomas [1].

The pathogenesis is not fully understood; it was proposed that de novo PC may develop from a pre-existing poroma through various triggers, including exposure to chemical agents, ultraviolet light, trauma, burning or radiotherapy, immunosuppressive drugs, especially after organ transplantation, and acquired immunodeficiency syndrome. It mainly occurs in adults aged between 21 to 90 years, according to different studies. PC occurs in both sexes equally [2-5].

Recent literature has reported that PC most commonly arises on the lower limbs (33\%), head and neck (32\%), trunk $(14.7 \%)$, genitalia/buttocks $(11.2 \%)$, and upper limbs $(7.4 \%)$. The location on the arms represents only $4.8 \%$ of PC common sites (only 14 cases have been registered at the moment) [6-16].

In this case report, we present a case of PC that gradually ulcerated over about 10 months on the left arm of a 93-year-old male patient with various comorbidities with a literature review on PC and its potential therapy.

\section{Case Presentation}

A 93-year-old Caucasian male patient came to our observation after undergoing resection of a grayish-yellow polypoid neoplasm with irregular contours and 2.5 -cm maximum diameter in the skin of the distal third of the left arm approximately 8 months ago.

Originally, the neoplasm was mobile, with a brownish-red color, an irregularly circular base, and a maximum diameter of less than one centimeter, and asymptomatic (Fig. 1). Following rapid growth, the patient underwent surgical excision of a skin and subcutis lozenge measuring $5.5 \times 2.3 \times 1.5 \mathrm{~cm}$ under local anesthesia with lidocaine. Histological examination revealed a largely ulcerated and necrotic PC infiltrating the papillary dermis and the first portion of the reticular dermis (Fig. 2). 
On clinical examination, the patient, transported in a wheelchair due to a severe degenerative joint disease limiting walking, had a very poor general condition, a ECOG (Eastern Cooperative Oncology Group) PS (performance status) of 3 , and declining edema without other skin lesions or palpable superficial lymphadenopathy.

The histological picture and clinical history made it possible to formulate the diagnosis of PC that probably arose on a pre-existing poroma. Ultrasound of the abdomen and main lymph nodes and a chest X-ray were normal, excluding metastases.

Considering the patient's age, poor general condition, comorbidities, and absent distant metastasis, periodic checks in the dermatological clinic were indicated. Written informed consent was obtained from the patient for publication of this case report and accompanying images.

\section{Discussion}

$\mathrm{PC}$ is a rare, aggressive, and chemoresistant skin cancer arising from one of the four primary adnexal structures of the skin: the hair follicle and sebaceous, eccrine, and apocrine glands; more exactly, it derives from terminal cells of the intraepithelial part of the eccrine duct called the acrosyringium [3]. It can arise de novo or from poroma ground with high potential for locoregional metastatic spread, morbidity, and mortality. It mostly occurs in elderly patients and the most common locations are the lower limbs, the trunk, and the head and neck.

This aggressive tumor can metastasize to local lymph nodes or distant organs. Skin metastases may be found near the primary tumor. Other less usual metastatic sites include lung, liver, breast, ovaries, bone, bladder, and retroperitoneum. The most common locations affected by PC are the lower extremities, head and neck, trunk, genitalia/buttocks, and upper extremities [4].

PCs located on the genitalia and buttocks have a higher propensity for distant metastases compared to those arising in the head and neck area. Metastatic disease is already present at diagnosis in up to $31 \%$ of cases, where the most common sites involved are regional lymph nodes (60\%) and lungs (13\%). The related prognosis is poor, with $50 \%-80 \%$ mortality and $9 \%$ of overall survival rates of 10 -years.

To date, only 15 cases occurring on the arms (including the current case) have been documented in the literature [6-16]. Clinically, PC presents as a slow-growing, red, dome-shaped skin nodule with a shiny surface or as a wart-like plaque, papule, or polyp that is sometimes painful and ulcerative. The lesion size can range from less than $1 \mathrm{~cm}$ to up to $10 \mathrm{~cm}$.

Due to its rarity, PC can mimic other skin conditions and could be misdiagnosed as other cutaneous neoplasms, particularly as squamous cell carcinoma. Differential diagnosis is mandatory in the presence of other skin lesions, such as amelanotic melanoma, seborrheic keratosis, pyogenic granuloma, Bowen's disease, verruca vulgaris, fibroma, or even metastatic adenocarcinoma. Thus, diagnosis is often delayed, and optimal management is lacking. Due to its aggressive nature, the prognosis for local recurrence after standard-wide local excision is poor, with a recurrence rate of $20 \%$ [7].

Histologically, PC can be recognized by lobules and cords of pleomorphic basaloid cells with enlarged hyperchromatic nuclei and mitotic activity. Two histopathological variants of PC exist: trabecular and epidermotropic. The second one is more aggressive than the first and is frequently associated with local recurrences and metastatic lesions [5].

The survival for patients with distant metastases is reported to be 5 to 24 months. Mortality is high, reaching $80 \%$ in distant diseases and $65 \%-67 \%$ when lymph nodes are involved. Given its rare occurrence, most of the literature has reported single clinical cases and a few case series, and there are currently no uniform international guidelines for these patients [6-16].

There is no standard treatment for metastatic disease. Undoubtedly, the treatment of choice is wide excision, which results in cure rates of $70 \%$ to $80 \%$. Although good results have been reported with 3- to 5 -mm resection margins, surgical margins should range from 1 to $2 \mathrm{~cm}$. When the disease is inoperable or advanced, systemic 
treatment represents a challenge. Mohs micrographic surgery may be a valid effective treatment option for PC. The role of sentinel lymph node biopsy is not yet well defined, although it could be useful for high-risk $\mathrm{PC}$ in cases with signs of histological aggression or intralymphatic involvement [7].

Treatment of distant metastatic PC is not yet well defined. Neither chemotherapy nor radiotherapy has been clinically advantageous in metastatic disease. In this regard, various therapeutic schemes have been evaluated with many uncertain results. Among these, taxane-based regimes have proven to be effective, with a response in $50 \%$ of cases. Paclitaxel plus cetuximab have been observed to provide an almost complete morphological response and a complete metabolic response at all locations of metastatic PC with strong epidermal growth factor receptor (EGF-R) expression, except for persistent bone metastases, where, however, metabolic activity was low due to the radiation therapy [17].

In the literature, we found other interesting case reports. Complete remission was also seen with polychemotherapy with doxorubicin, mitomycin C, vincristine, and 5-fluorouracil (5-FU), alternating with cisplatin and bleomycin or hyperthermic perfusion with 5-FU and melphalan. Other agents have been administered in combination, including cisplatin and docetaxel, carboplatin and paclitaxel; doxorubicin, mitomycin, vincristine, and 5-fluorouracil; anthracycline, cyclophosphamide, vincristine, and bleomycin; isotretinoin and interferon-alpha [18]. Other case series described using topical 5-FU and intraarterial docetaxel and docetaxel as a single agent. Furthermore, long-term outcomes have been recently achieved with pembrolizumab [19].

Radiosensitivity of PC is suggested by some case reports. Adjuvant radiotherapy is not yet standard of care, but it would be recommended in high-risk situations, such as cases with positive surgical margins, tumors larger than $5 \mathrm{~cm}$, lymphovascular involvement, and tumors with moderate to poorly differentiated grading [20]. Metastatic cervical lymph nodes successfully treated with cyberknife radiosurgery have also been mentioned.

In conclusion, the best chance for cure is early diagnosis and surgery because neither chemotherapy nor radiotherapy has shown some clinical benefit in metastatic PC.

\section{Conclusions}

$\mathrm{PC}$ is rare and challenging to recognize skin cancer with a high risk of recurrence and metastasis. Despite its rarity, eccrine $\mathrm{PC}$ is the most common sweat gland. The differential diagnosis for every suspicious cutaneous lesion is needed to prevent delays in treatment.

Our clinical case suggests that early identification and complete excision offer the best chances of feasible recovery even in elderly patients in poor general condition and with numerous comorbidities.

No treatment has been standardized yet. Surgery is the first and only therapeutic option that could significantly affect patient outcomes. Chemotherapy and radiotherapy have not shown clinical benefits in the management of advanced PC.

Due to its rarity, further research, including case reports, is necessary to improve the diagnosis and therapy of PC.

\section{Authors' contributions.}

Conceptualization: CS. Data Curation: CS. Investigation: CS EMR LZC VR AP. Visualization: CS VR. Writing - Original Draft Preparation: CS. Writing - Review \& Editing: CS EMR. All authors have read and approved the final manuscript.

\section{References}

1. Pinkus H, Mehregan AH. Epidermotropic eccrine carcinoma. A case combining features of eccrine poroma and Paget's dermatosis. Arch Dermatol. 1963 Nov;88:597-606. doi: 10.1001/archderm.1963.01590230105015 
2. Mehregan AH, Hashimoto K, Rahbari H. Eccrine adenocarcinoma. A clinicopathologic study of 35 cases. Arch Dermatol. 1983 Feb;119(2):104-14. doi: 10.1001/archderm.119.2.104

3. Robson A, Greene J, Ansari N, et al. Eccrine porocarcinoma (malignant eccrine poroma): a clinicopathologic study of 69 cases. Am J Surg Pathol. 2001 Jun;25(6):710-20. doi: 10.1097/00000478-20010600000002

4. Salih AM, Kakamad FH, Baba HO, et al. Porocarcinoma; presentation and management, a metaanalysis of 453 cases. Ann Med Surg (Lond). 2017 Jun 20;20:74-79. doi: 10.1016/j.amsu.2017.06.027

5. Idrissi Serhrouchni K, Harmouch T, Chbani L, et al. Eccrine carcinoma : a rare cutaneous neoplasm. Diagn Pathol. 2013 Feb 4;8:15. doi: 10.1186/1746-1596-8-15

6. Mitchell DC, Kuehn GJ, Scott GA, et al. A Rare Case of Porocarcinoma and Trichoblastoma Arising in a Nevus Sebaceus of Jadassohn. Case Rep Dermatol Med. 2021 Mar 3;2021:7598086. doi: $10.1155 / 2021 / 7598086$

7. Le HML, Faugeras L, De Moor V, et al. Eccrine Porocarcinoma: A Challenging Diagnostic and Therapeutic Tumoral Entity. Case Rep Oncol. 2021 May 10;14(2):700-705. doi: 10.1159/000514984

8. van den Brand AC, Damman J, Groenendijk FH, Waalboer-Spuij R. Eccrine porocarcinoma: A rare case of an in situ tumor with lymph node metastases. JAAD Case Rep. 2019 Dec 24;6(1):42-45. doi: 10.1016/j.jdcr.2019.10.020.

9. Fujimine-Sato A, Toyoshima M, Shigeta S, et al. Eccrine porocarcinoma of the vulva: a case report and review of the literature. J Med Case Rep. 2016 Nov 10;10(1):319. doi: 10.1186/s13256-016-1106-1

10. Chang O, Elnawawi A, Rimpel B, et al. Eccrine porocarcinoma of the lower extremity: a case report and review of literature. World J Surg Oncol. 2011 Aug 22;9:94. doi: 10.1186/1477-7819-9-94

11. Seo BF, Choi HJ, Jung SN. Eccrine porocarcinoma on the cheek. Arch Craniofac Surg. 2019 Feb;20(1):48-50. doi: 10.7181/acfs.2018.02180 Epub 2019 Feb 11

12. Nazemi A, Higgins S, Swift R, In G, Miller K, Wysong A. Eccrine Porocarcinoma: New Insights and a Systematic Review of the Literature. Dermatol Surg. 2018 Oct;44(10):1247-1261. doi: 10.1097/DSS.0000000000001566

13. Oh S, Behzadnia A, Chan J. Eccrine Porocarcinoma: A Case Report of a Rare and Aggressive Cutaneous Tumour. Cureus. 2019 Nov 27;11(11):e6244. doi: 10.7759/cureus.6244

14. Grieco M, Simonacci F, Grignaffini E, et al. Eccrine porocarcinoma: case report and review of the literature. G Ital Dermatol Venereol. 2020 Aug;155(4):500-504. doi: 10.23736/S0392-0488.17.05182-3

15. Rafiei R, Eftekhari H, Daryakar A, et al. Eccrine porocarcinoma: a case report and brief review of the literature. Our Dermatol Online. 2016;7(4):391-393. DOI:10.7241/ourd.20164.106

16. Charles NC, Kim ET. Porocarcinoma of the Eyelid Presenting as an Umbilicated Lesion. Ophthalmic Plast Reconstr Surg. 2021 Nov-Dec 01;37(6):e224. doi: 10.1097/IOP.0000000000001956

17. Godillot C, Boulinguez S, Riffaud L, et al. Complete response of a metastatic porocarcinoma treated with paclitaxel, cetuximab and radiotherapy. Eur J Cancer. 2018 Feb;90:142-145. doi: 10.1016/j.ejca.2017.11.009 Epub 2017 Dec 9

18. Płachta I, Kleibert M, Czarnecka AM, et al. Current Diagnosis and Treatment Options for Cutaneous Adnexal Neoplasms with Apocrine and Eccrine Differentiation. Int J Mol Sci. 2021 May 11;22(10):5077. doi: $10.3390 /$ ijms22105077

19. Lee KA, Cioni M, Robson A, Bataille V. Metastatic porocarcinoma achieving complete radiological and clinical response with pembrolizumab. BMJ Case Rep. 2019 Sep 5;12(9):e228917. doi: 10.1136/bcr2018-228917

20. Kim HJ, Kim A, Moon KC, et al. Eccrine Porocarcinoma: A Multicenter Retrospective Study with Review of the Literatures Reported in Korea. Ann Dermatol. 2020 Jun;32(3):223-229. doi: 10.5021/ad.2020.32.3.223 Epub 2020 Apr 24

Figures 


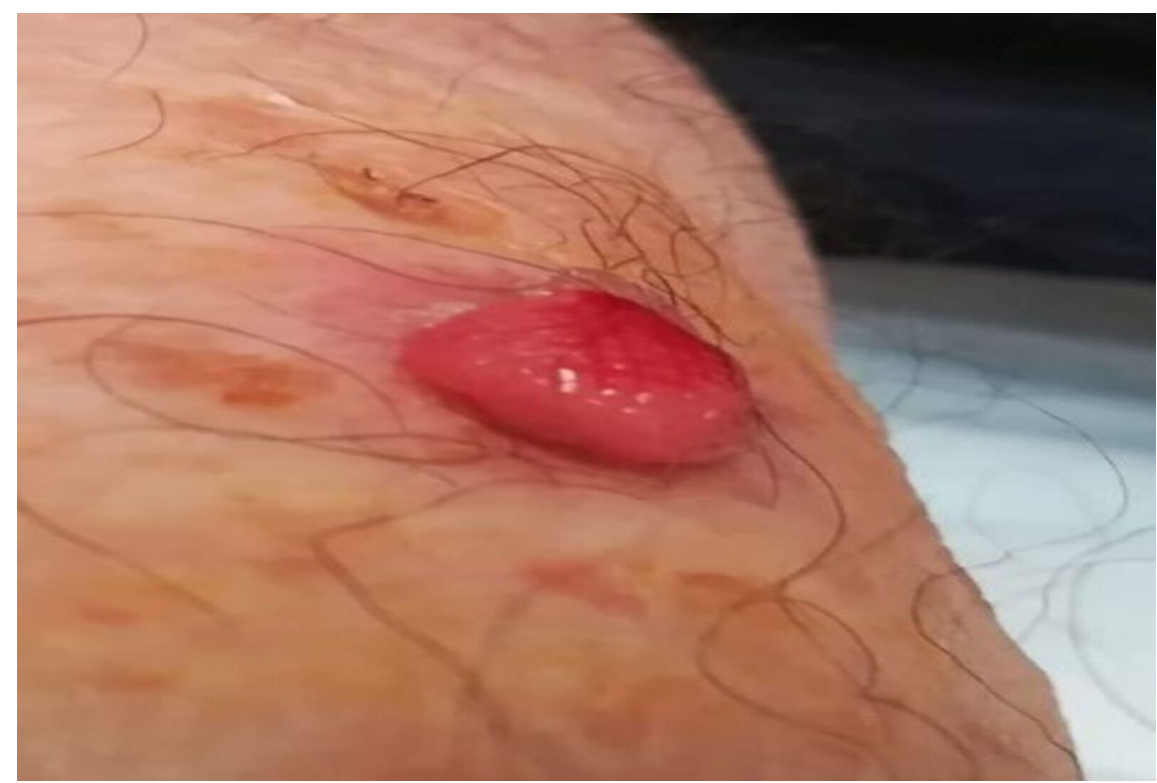

Fig. 1 Porocarcinoma of the left arm.

Clinical appearance of the $2.5 \mathrm{~cm}$ of maximum diameter brownish-red color polypoid neoformation with irregular contours and circular base arising at the skin level of the distal third of the left arm.

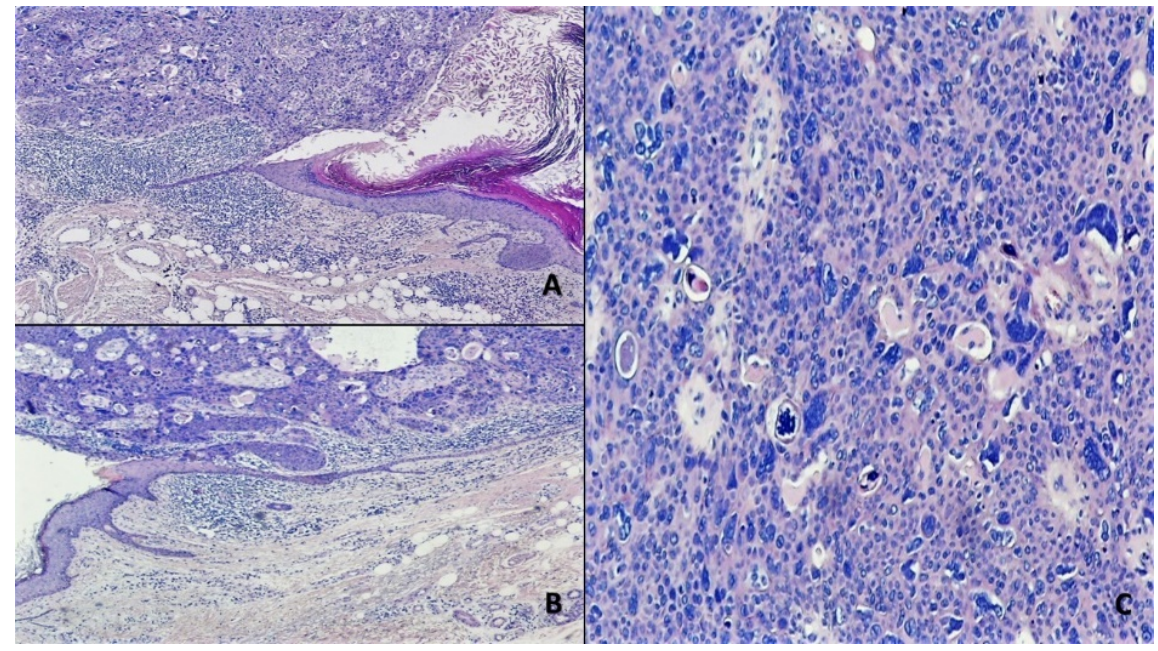

Fig. 2 Photomicrograph of histopathology of the resected tumor.

(A, B) Hematoxylin eosin stain (25x): invasive porocarcinoma displays an intradermal proliferation with infiltrative borders and atypical cytological features; (C) Hematoxylin eosin stain (40x): higher magnification with marked cytonuclear atypia. 

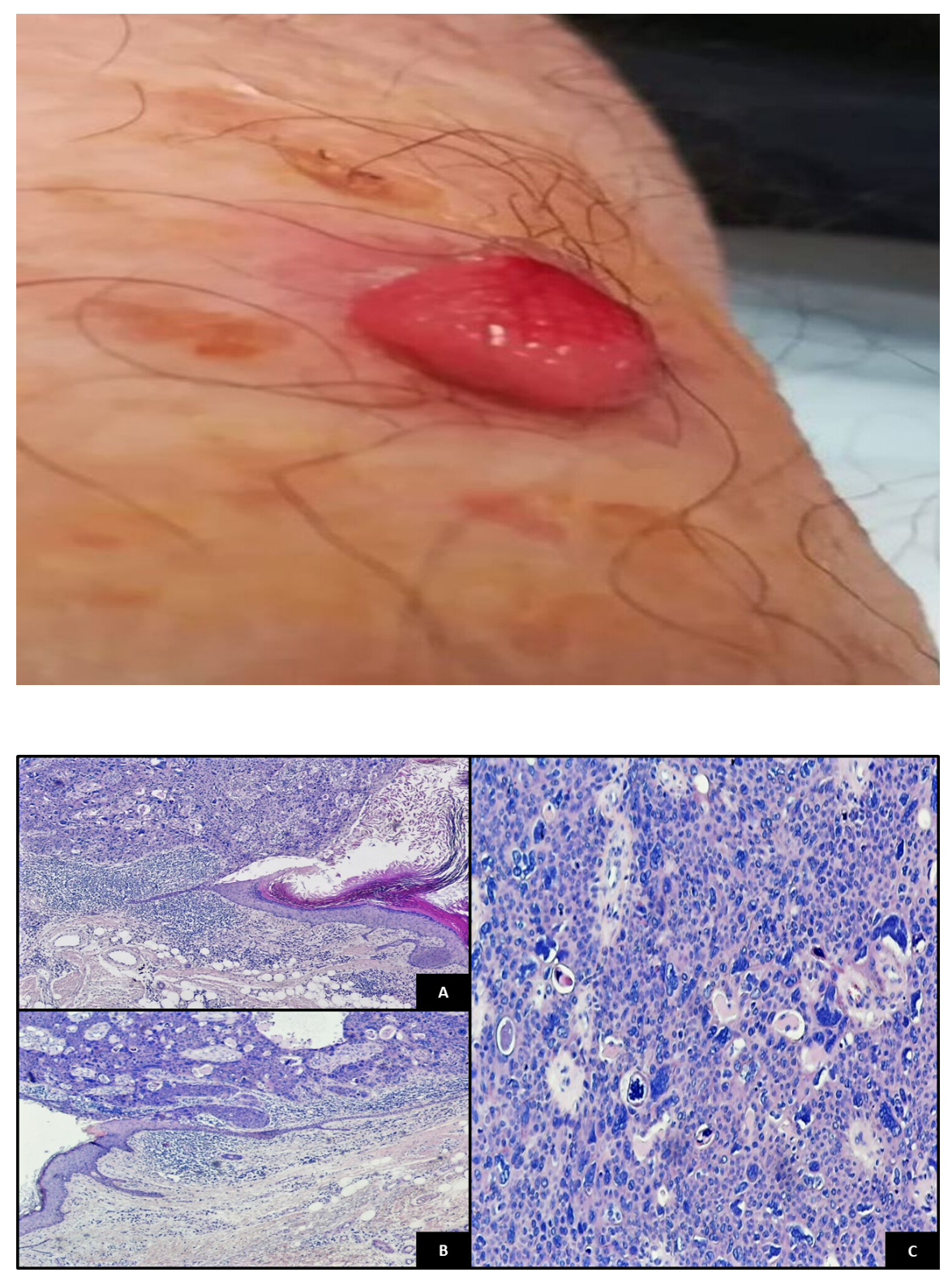